\author{
Mathematical Sciences And Applications E-Notes \\ Volume 3 No. 1 Pp. 1-12 (2015) C MSAEN
}

\title{
DEGREES OF SOLID VARIETIES OF SEMIRINGS
}

\author{
HIPPOLYTE HOUNNON AND KLAUS DENECKE
}

(Communicated by İrfan ŞİAP)

\begin{abstract}
For any arbitrary variety $V$, the degree $d_{p}(V)$ of $V$ with respect to proper hypersubstitutions was introduced in [6]. This degree of any variety of bands was determined in [4]. In this paper we characterize the universe of the free algebra of each solid variety of semirings and from this we derive the degree $d_{p}(V)$ if $V$ is any solid variety of semirings.
\end{abstract}

\section{INTRODUCTION}

Hypersubstitutions are mappings sending operation symbols to terms and preserving arities. They can be applied to algebras and equations. This gives the concepts of a derived algebra and of a hyperidentity. If every identity of a given variety $V$ of algebras is a hyperidentity, then the variety $V$ is said to be solid. More details on the notions of hypersubstitions, hyperidentities and solid varieties can be seen in [9], [8] and [7]. All solid varieties of semirings were determined (see in [3]). Now, we want to investigate some properties of solid varieties of semirings. In Universal Algebra like also in other parts of Mathematics, numerical invariants play an important role. An example of a numerical invariant in Universal Algebra is the spectrum of a variety $V$ which is defined as a sequence $\operatorname{spect}(V):=\left(\left|\mathcal{F}_{V}(n)\right|\right)_{n \geq 1}$ of the cardinality of the $n$-generated free algebras with respect to $V$. In this paper, we consider a numerical invariant related to the spectrum, the degree of a variety. A hypersubstitution is called $V$-proper if it preserves all identities of the variety $V$. The set of all $V$-proper hypersubstitions will be denoted by $P(V)$.

The degree $d_{p}(V)$ of the variety $V$ with respect to proper hypersubstitutions is the cardinality of the quotient set $\left.P(V)\right|_{\sim_{V}}$, where $\sim_{V}$ is the binary relation on the set $\operatorname{Hyp}(\tau)$ (of all hypersubstitutions of type $\tau=\left(n_{i}\right)_{i \in I}$ ), introduced in [11] and defined by $\sim_{V}: \sigma_{1} \sim_{V} \sigma_{2}$ iff $\sigma_{1}\left(f_{i}\right) \approx \sigma_{2}\left(f_{i}\right) \in I d V$ for $i \in I$.

Then one can ask the following questions:

- What is the degree of a given variety?

Date: Received: May 19, 2013; Revised: August 29, 2014; Accepted: November 25, 2014.

2010 Mathematics Subject Classification. MSC2010 16Y60, 03C05,08C85.

Key words and phrases. Semiring, hypersubstitution, hyperidentity, solid variety.

The authors are greatful to the referee for his valuable comments. The first author is also greatful to the financial support of Benin government within the framework of the program of the lecturer's training. 
- For a given integer, determine all varieties which have this integer as its degree. - What structural properties of a given variety $V$ can be derived from informations about the degree?

Some of these questions are already answered for varieties of semigroups. For instance, $d_{p}(V)$ is known for any variety $V$ of bands (idempotent semigroups) and in this case $d_{p}(V) \in\{1,2,3,4,6\}$ (see in [4] and [13]).

In this paper, we want to answer the first question in the case of solid varieties of semirings. To do this we need to recall some basic concepts.

1. A semiring is a non-empty set $S$ together with two binary operations + and $\cdot$ such that the algebras $(S ;+)$ and $(S ; \cdot)$ are semigroups which are related by the distributive identities:

$$
x_{1}\left(x_{2}+x_{3}\right) \approx x_{1} x_{2}+x_{1} x_{3} \text { and }\left(x_{1}+x_{2}\right) x_{3} \approx x_{1} x_{3}+x_{2} x_{3} .
$$

The variety of all semirings will be denoted by $S R$.

2. A semiring $(S ;+, \cdot)$ is medial if

$$
\begin{gathered}
x_{1}+x_{2}+x_{3}+x_{4} \approx x_{1}+x_{3}+x_{2}+x_{4} \in I d S \text { and } \\
x_{1} x_{2} x_{3} x_{4} \approx x_{1} x_{3} x_{2} x_{4} \in I d S .
\end{gathered}
$$

( $I d S$ is the set of all identities satisfied in the algebra $(S ;+, \cdot)$ ).

3. A semiring $(S ;+, \cdot)$ is idempotent if

$$
x_{1}+x_{1} \approx x_{1} \approx x_{1} \cdot x_{1} \in I d S .
$$

4. A semiring $(S ;+, \cdot)$ is distributive if

$$
\begin{gathered}
x_{1} x_{2}+x_{3} \approx\left(x_{1}+x_{3}\right)\left(x_{2}+x_{3}\right) \in I d S \text { and } \\
x_{1}+x_{2} x_{3} \approx\left(x_{1}+x_{2}\right)\left(x_{1}+x_{3}\right) \in I d S .
\end{gathered}
$$

5. A variety $V$ of semirings is medial if all algebras in $V$ are medial. In a similar way one can define the varieties of distributive semirings and idempotent semirings, respectively.

6. An equation $s \approx t$ is regular if both terms $s, t$ contain the same variables. A variety $V$ is regular if all identities in $V$ are regular.

It is well known that a variety $V$ is regular iff its basis identities are regular.

If $V$ is a variety of semirings and $\Sigma$ is a set of equations, by $V(\Sigma)$ we denote the subvariety of $V$ which is generated by the set $\Sigma$. For reference, below we list some varieties to be used in this paper:

$V_{M I D}$ - the variety of all medial, idempotent and distributive semirings,

$V_{B E}:=V_{M I D}\left(\left\{\left(x_{1}+x_{2}\right)\left(x_{2}+x_{1}\right) \approx x_{1} x_{2}+x_{2} x_{1}\right\}\right)$

$R A_{2,2}:=S R\left(\left\{x_{1}+x_{2}+x_{3} \approx x_{1}+x_{3}, x_{1} x_{2} x_{3} \approx x_{1} x_{3}, x_{1} x_{1} \approx x_{1} \approx x_{1}+x_{1}\right\}\right) ;$

$\mathcal{T}$ - the trivial variety of semirings, i.e. the class of all one-element semirings.

It is clear that the varieties $V_{M I D}$ and $V_{B E}$ are regular since their basis identities are regular.

Now, we turn to the theory of hyperidentities.

Let $W_{(2,2)}\left(X_{2}\right)$ be the set of all binary terms of type $(2,2)$ built up by variables from the alphabet $X_{2}=\{x, y\}$. The operation symbols $F$ and $G$ will be denoted sometimes additively and multiplicatively, respectively.

Hypersubstitutions of type $\tau=(2,2)$ are mappings

$$
\sigma:\{F, G\} \rightarrow W_{(2,2)}\left(X_{2}\right)
$$


A hypersubstitution $\sigma$ of type $(2,2)$ can be extended to a mapping $\hat{\sigma}$ defined on the set $W_{(2,2)}(X)$ of all terms of type $(2,2)$, where $X=\{x, y, z, u, v, \cdots\}$ is an arbitrary countably infinite alphabet of variables, by the following steps:

(i) $\hat{\sigma}[t]:=t$, if $t \in X$ is a variable, and

(ii) $\hat{\sigma}\left[f\left(t_{1}, t_{2}\right)\right]:=\sigma(f)\left(\hat{\sigma}\left[t_{1}\right], \hat{\sigma}\left[t_{2}\right]\right), f \in\{F, G\}$ for composed terms.

The right hand side of (ii) must be interpreted as a superposition of term operations of the term algebra of type $(2,2)$.

The hypersubstitution $\sigma$ of type $(2,2)$ such that $\sigma(F)=t$ and $\sigma(G)=s$ will be denoted by $\sigma_{t, s}$. Together with the hypersubstitution $\sigma_{i d}$ defined by

$\sigma_{i d}(f)=f(x, y), f \in\{F, G\}$, the set of all hypersubstitutions of type $(2,2)$ forms a monoid, denoted by Hyp.

An identity $s \approx t$ in a variety $V$ of semirings is called a hyperidentity in $V$ if for every $\sigma \in H y p$ the equations $\hat{\sigma}[s] \approx \hat{\sigma}[t]$ belong to the set $I d V$ of all identities satisfied in $V$.

A variety $V$ is called solid if all identities in $V$ are satisfied as hyperidentities.

Let $V$ be a variety. A hypersubstitution $\sigma$ is called $V$-proper (or preserving all identities) if for all identities $s \approx t \in I d V, \hat{\sigma}[s] \approx \hat{\sigma}[t] \in I d V$.

Now, we will determine the universe of the free algebra with respect to each element of the lattice of all solid varieties of semirings. This lattice was fully described in [3] as follows:

Theorem 1.1. [3] The lattice of all solid varieties of semirings is the 4-element chain $\mathcal{T} \subset R A_{(2,2)} \subset V_{B E} \subset V_{M I D}$.

\section{Free algebras with Respect to Solid Varieties of Semirings}

In this section we will determine the free algebra with respect to each non-trivial solid variety of semirings.

Definition 2.1. [12] The term algebra of type $\tau=\left(n_{i}\right)_{i \in I}$ is the algebra $\mathcal{F}_{\tau}(X):=\left(W_{\tau}(X) ;\left(\overline{f_{i}}\right)_{i \in I}\right)$, where

$\overline{f_{i}}\left(t_{1}, \ldots, t_{n_{i}}\right):=f_{i}\left(t_{1}, \ldots, t_{n_{i}}\right)$ for all $t_{1}, \ldots, t_{n_{i}} \in W_{\tau}(X)$ and for all $i \in I$.

For every variety $V$, it is clear that $I d V$ is a congruence relation on the term algebra $\mathcal{F}_{\tau}(X)$. Then we can construct the quotient algebra $\mathcal{F}_{\tau}(X) / I d V$ which is called free algebra with respect to $V$ freely generated by the set $X$ and is denoted by $\mathcal{F}_{V}(X)$. The free algebra with respect to $V$ freely generated by the set $X_{n}=\left\{x_{1}, \ldots, x_{n}\right\}$ will be denoted by $\mathcal{F}_{V}(n)$.

The following results and concepts are needed.

Lemma 2.1. [3] Every subvariety $V$ of $V_{M I D}$ satisfies the following identities:
1. $x+x y+y \approx x+y$,
10. $x y+y x y+y x \approx x y+y x$,
2. $x+y x+y \approx x+y$,
11. $x y+y x y+y \approx x y+y$,
3. $x+x y x+x y \approx x+x y$,
12. $x+x y x+y \approx x+y$,
4. $x+x y x+y x \approx x+y x$,
13. $x+y x y+y \approx x+y$,
5. $x+y x+y x y \approx x+y x y$,
14. $x y x+y x+y \approx x y x+y$,
6. $x+x y+y x y \approx x+y x y$,
15. $x y x+x y+y \approx x y x+y$,
7. $x+x y x+y x y \approx x+y x y$,
8. $x y+x y x+x \approx x y+x$,
16. $x y x+x y+y x y \approx x y x+y x y$,
9. $x y+x y x+y x \approx x y+y x$,
17. $x y x+y x+y x y \approx x y x+y x y$,
18. $x y x+y x y+y \approx x y x+y$. 
Definition 2.2. (1) Let $V$ be a variety. Two terms $t$ and $s$ are called $V$ equivalent if $s \approx t \in I d V$ (see in [10]).

(2) An equation $s \approx t$ is outermost, if the terms $s$ and $t$ start with the same variable and end also with the same variable. A variety $V$ is called outermost, if all identities of $I d V$ are outermost (see in [8]).

It is well known that a variety is outermost iff its basis identities are outermost. Let $\operatorname{Bin}_{G}=\{x, y, x y, y x, x y x, y x y\}$. It is clear that two different elements of Bin $_{G}$ are not $V$-equivalent for $V=V_{M I D}$ or $V=V_{B E}$ because $V_{M I D}$ and $V_{B E}$ are outermost and regular (since the basis identities of $V_{M I D}$ and $V_{B E}$ are outermost and regular).

Lemma 2.2. [3] Let $V$ be a subvariety of $V_{M I D}$. Then every binary term $t$ is $V$-equivalent to a sum of the form $t_{1}+t_{2}+t_{3}+t_{4}$, where $t_{i} \in \operatorname{Bin}_{G}, i=1,2,3,4$.

Now, we can prove:

Lemma 2.3. Let $s_{i}, t_{i}, i \in\{1,2,3,4\}$ be elements from Bin . $_{\text {. }}$ If $s_{1}+s_{2}+s_{3}+s_{4} \approx t_{1}+t_{2}+t_{3}+t_{4} \in I d V$, with $V \in\left\{V_{M I D}, V_{B E}\right\}$ then $s_{1}=t_{1}$ and $s_{4}=t_{4}$.

Proof: Since $V$ is solid, applying the hypersubstitutions $\sigma_{x, x y}$ (resp. $\sigma_{y, x y}$ ) to the identity $s_{1}+s_{2}+s_{3}+s_{4} \approx t_{1}+t_{2}+t_{3}+t_{4} \in I d V$, where $s_{i}, t_{i} \in \operatorname{Bin}_{G},(i \in\{1,2,3,4\})$, we obtain in $V$ the identity $s_{1} \approx t_{1}$ (resp. $s_{4} \approx t_{4}$ ). This leads to the equalities $s_{1}=t_{1}$ and $s_{4}=t_{4}$ since the binary terms $t_{i}, s_{i}, i=1,2,3,4$ belong to $\operatorname{Bin}_{G}$ and two different elements of $\operatorname{Bin}_{G}$ are not $V$-equivalents.

The following lemma gives another generating identity of $V_{B E}$.

Lemma 2.4. [3] The variety $V_{M I D}(x y+x y x+x y \approx x y+x+x y)$ is equal to the variety $V_{B E}$.

Using the previous lemma, we have:

Lemma 2.5. (1) The identities $x y x+x+x y x \approx x y x$ and $x y+y x \approx x y+t+y x$, for $t \in\{x, y\}$ hold in $V_{B E}$ but not in $V_{M I D}$.

(2) Let $u \in\{x, y\}$ and $t_{i} \in \operatorname{Bin}_{G} \backslash\{u\}, i=1,2,3$. Then $t_{1}+t_{2}+t_{3} \approx t_{1}+u+t_{3} \notin I d V_{M I D}$.

\section{Proof:}

(1) From $x y+y x \approx x y+x+y+y x \in I d V_{B E}$, it follows the identity $x y x \approx x y x+$ $x+x y x \in I d V_{B E}$ by substituting $x y x$ for $y$ and using the idempotency and the medial laws. Moreover, the variety $V_{B E}$ satisfies the following identities: $x y+x+y x \approx x y+y x+x+y x \approx x y+x+y+y x \approx x y+y x \approx x y+y+y x$, using the idempotency, the medial law and the identity $x y+x+y+y x \approx$ $x y+y x \in I d V_{B E}$.

Assume that the variety $V_{M I D}$ satisfies the identity $x y x+x+x y x \approx x y x$. Then we would get in $V_{M I D}$ the identities

$x y+x y x+x y \approx x y+x y x+x+x y x+x y \approx x y+x+x y$ by Lemma $2.1(3)$. This leads to the equalities $V_{M I D}=V_{M I D}(x y+x y x+x y \approx x y+x+x y)=$ $V_{B E}$ (Lemma 2.4). This contradicts $V_{M I D} \neq V_{B E}$.

Assume that the identity $x y+x+y x \approx x y+y x$ or the identity $x y+y+$ 
$y x \approx x y+y x$ holds in the variety $V_{M I D}$. Then substituting $x y x$ for $y$ in the first identity and $y x y$ for $y$ in the second one, leads to the equation $x y x+x+x y x \approx x y x$ which is not satisfied as an identity in $V_{M I D}$.

(2) Let $u, v \in\{x, y\}$ such that $u \neq v$ and $t_{i} \in \operatorname{Bin}_{G} \backslash\{u\}, i=1,2,3$.

Assume that $t_{1}+t_{2}+t_{3} \approx t_{1}+u+t_{3} \in I d V_{M I D}$.

The substitution $v$ by $u v u$ in $t_{1}+t_{2}+t_{3} \approx t_{1}+u+t_{3} \in I d V_{M I D}$ gives $u v u \approx u v u+u+u v u \in I d V_{M I D}$. This contracdicts 1 .

Lemma 2.6. Let $\left(t_{1}, t_{2}\right) \in(\{x, x y, x y x\})^{2}$ such that the equation $t_{1} \approx t_{2}$ is outermost. Let $(s, t) \in\left(\operatorname{Bin}_{G}\right)^{2}$. If the equation $s \approx t$ is not outermost, then $t_{1}+s+t_{2} \approx t_{1}+t+t_{2} \notin I d V_{B E}$.

Proof: Assume that $t_{1}+s+t_{2} \approx t_{1}+t+t_{2} \in I d V_{B E}$. Since the equation $s \approx t$ is not outermost, without loss of generality, we can assume that $s$ starts with $x$ and $t$ with $y$. Applying $\sigma_{x+y, x}$ to the equation $t_{1}+s+t_{2} \approx t_{1}+t+t_{2}$ gives (using the idempotency) the equation $x \approx x+y+x$ which is not satisfied as identity in $V_{B E}$ because $V_{B E}$ is regular.

Lemma 2.7. There are exactly (up to permutation of variables $x$ and $y$ ) the following binary terms which are $V_{M I D}$-equivalent to sums consisting of three summands from Bin $_{G}$, these sums cannot be $V_{M I D}$-equivalent to sums consisting of at most two summands from Bin $_{G}$ :
1. $x+x y+t, t \in\{x, x y x, y x\}$,
2. $x+y+t, t \in \operatorname{Bin}_{G} \backslash\{y\}$,
3. $x+y x+t, t \in\{x, x y x, x y\}$,
9. $x y x+y x y+t, t \in\{x, x y x\}$,
10. $x y x+y x+t, t \in\{x, x y, x y x\}$
4. $x+y x y+t, t \in\{x, x y x\}$,
11. $x y+x+t, t \in \operatorname{Bin}_{G} \backslash\{x\}$,
5. $x+x y x+x$,
6. $x y x+x+t, t \in \operatorname{Bin}_{G} \backslash\{x\}$,
12. $x y+y x+t, t \in \operatorname{Bin}_{G} \backslash\{y x\}$,
13. $x y+y+t, t \in \operatorname{Bin}_{G} \backslash\{y\}$,
7. $x y x+y+t, t \in \operatorname{Bin}_{G} \backslash\{y\}$,
8. $x y x+x y+t, t \in\{x, y x, x y x\}$,
14. $x y+x y x+x y$,
15. $x y+y x y+x y$.

Proof: To prove that $s \approx t \notin I d V_{M I D}$, we will show sometimes that $s \approx t \notin I d V_{B E}$, since $V_{B E} \subset V_{M I D}$ so $I d V_{M I D} \subseteq I d V_{B E}$.

I) First, we show that each term in the list of Lemma 2.7 cannot be $V_{M I D}$-equivalent to a sum of at most two elements from $\operatorname{Bin}_{G}$.

- To prove that each term in the list (Lemma 2.7) cannot be $V_{M I D}$-equivalent to an element from $\operatorname{Bin}_{G}$, using Lemma 2.3, we have to consider the equations $x+t_{1}+x \approx x, t_{1} \in \operatorname{Bin}_{G} \backslash\{x\} ; x y+t_{2}+x y \approx x y, t_{2} \in \operatorname{Bin}_{G} \backslash\{x y\}$ $x y x+t_{3}+x \approx x y x, t_{3} \in B_{i n} \backslash\{x y x\}$; and to show that the variety $V_{M I D}$ does not satisfy one of them as identities.

Indeed, the idempotency and Lemma 2.6 ensure that none of the identities $\left.x+t_{1}+x \approx x, t_{1} \in \operatorname{Bin}_{G} \backslash\{x, x y x\} ; x y+t_{2}+x y \approx x y, t_{2} \in B \operatorname{Bin}_{G} \backslash\{x y\}\right) ;$ $x y x+t_{3}+x \approx x y x, t_{3} \in \operatorname{Bin}_{G} \backslash\{x y x, x\}$ is satisfied in $V_{B E}$. The regularity property of $V_{B E}$ guaranties that $x+x y x+x \approx x \notin I d V_{B E}$.

We conclude that $x y x+x+x y x \approx x y x \notin I d V_{M I D}$ [see Lemma 2.5 (1)].

- Now, we prove that none of the terms in the list of Lemma 2.7 can be

$V_{M I D}$-equivalent to a sum consisting of two terms of $\operatorname{Bin}_{G}$.

Lemma 2.3, the idempotency and Lemma 2.6 ensure that we have to consider only 
the equations:

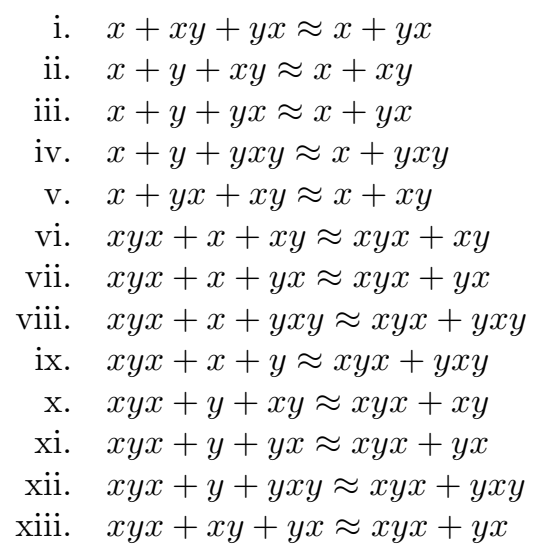

$$
\begin{aligned}
\text { xiv. } & x y x+y x+x y \approx x y x+x y \\
\text { xv. } & x y+x+y \approx x y+y \\
\text { xvi. } & x y+x+y x \approx x y+y x \\
\text { xvii. } & x y+x+x y x \approx x y+x y x \\
\text { xviii. } & x y+x+y x y \approx x y+y x y \\
\text { xix. } & x y+y x+x \approx x y+x \\
\text { xx. } & x y+y x+y \approx x y+y \\
\text { xxi. } & x y+y x+x y x \approx x y+x y x \\
\text { xxii. } & x y+y x+y x y \approx x y+y x y \\
\text { xxiii. } & x y+y+x \approx x y+x \\
\text { xxiv. } & x y+y+y x \approx x y+y x \\
\text { xxv. } & x y+y+x y x \approx x y+x y x \\
\text { xxvi. } & x y+y+y x y \approx x y+y x y
\end{aligned}
$$

and to show that the variety $V_{M I D}$ satisfies none of them as identities.

Assume that the previous identities (except iv.,vi., vii., viii.,ix., xii., xvi., xvii.,xxiv., xxvi.) are satisfied in $V_{B E}$.

Since $V_{B E}$ is solid, using the hypersubstitution $\sigma_{x+y, x}$ or $\sigma_{x+y, y}$, we will get the contradiction $x+y+x \approx x \in I d V_{B E}$. Therefore, we conclude that none of the identities (i.,ii.,iii. v., x.,xi.,xiii., xiv., xv., xviii.,xix.,xx., xxi., xxii.,xxiii. and xxv.) is satisfied in $V_{B E}$.

Lemma 2.5 (2) guarantees that none of the identities (iv.,vi., vii., viii.,ix., xii., xvi., xvii.,xxiv. and xxvi.) is satisfied in $V_{B E}$.

II) Secondly, we will prove that each term in the list is $V_{M I D}$-equivalent to itself only.

Using Lemma 2.3 and Lemma 2.6, we have to consider only the equation $t_{1}+t_{2}+t_{3} \approx$ $t_{1}+t_{4}+t_{3}$ (where both binary terms $t_{1}+t_{2}+t_{3}$ and $t_{1}+t_{4}+t_{3}$ belong to the list of Lemma 2.7, and the terms $t_{i}, i=1, \cdots, 4$ do not satisfy the conditions of Lemma $2.6)$ and to show that the variety $V_{M I D}$ does not satisfy one of them as identity.

1. We consider only the equation $x+x y+y x \approx x+y+y x$.

By Lemma $2.5(2)$, one has $x+x y+y x \approx x+y+y x \notin I d V_{M I D}$.

2. We consider the equations $x+y+x \approx x+y x y+x, x+y+x y \approx x+y x+$ $x y$, and $x+y+x y x \approx x+y x y+x y x$.

By Lemma $2.5(2)$, none of these identities holds in $V_{M I D}$.

3. There is no equation to consider.

4. and 5 . are similar to 3 .

6. We assume that the following identities hold in $V_{B E}$ :
a. $x y x+x+x y \approx x y x+y+x y$
b. $x y x+x+x y \approx x y x+y x+x y$
c. $x y x+x+y x \approx x y x+y+y x$
d. $x y x+x+y x \approx x y x+x y+y x$
e. $x y x+x+y x y \approx x y x+y+y x y$

Lemma 2.5 (2) shows that none of the above mentioned identities is satisfied in $V_{M I D}$.

7. can be proved in a similar way as in part 6 .

8.,9. and 10. are similar to 3 .

11. We have to consider the equations
a. $x y+x+y \approx x y+y x+y$
b. $x y+x+x y \approx x y+x y x+x y$
c. $x y+x+y x \approx x y+y+y x$
d. $x y+x+x y x \approx x y+y x+x y x$
e. $x y+x+x y x \approx x y+y+x y x$
f. $x y+x+y x y \approx x y+y+y x y$. 
Lemma 2.5 (2) guarantees that none of the identities a.), b.),c.),e.),f.) is satisfied in $V_{M I D}$.

Assume that the identity d.) holds in $V_{B E}$. Since $V_{B E}$ is solid, applying the hypersubstitution $\sigma_{x+y, y}$ to d.) we will obtain $y+x+y \approx y \in I d V_{B E}$. This contradicts the regularity property of $V_{B E}$.

12. is similar to 2 .

13. is similar to 2 .

14. Nothing to prove.

III) It is left to prove that any binary term that is a sum of three different elements from $\operatorname{Bin}_{G}$, is $V_{M I D}$-equivalent to one of the terms in our list. This was done in [10].

Lemma 2.8. There are exactly (up to permutation of variables $x$ and $y$ ) the following binary terms which are $V_{M I D}$-equivalent to sums consisting of four different elements from Bin $_{G}$, these sums cannot be $V_{M I D}$-equivalent to sums consisting of at most three summands from Bin $_{G}$ :

$$
\begin{array}{ll}
\text { 1. } x y x+x+y+t, t \in \operatorname{Bin}_{G} \backslash\{x, y\} & \text { 5. } x y+x+y x+t, t \in\{x y, x y x\} \\
\text { 2. } x y x+x+x y+t, t \in\{x y x, y x\} & \text { 6. } x y+y x+y+t \in\{x y, y x y\} \\
\text { 3. } x y x+x+y x+t, t \in\{x y x, x y\} & \text { 7. } x y+x+y+t, t \in B i n_{G} \backslash\{x, y\} . \\
\text { 4. } x y x+x+y x y+x y x &
\end{array}
$$

Proof: In the following lines, the idempotency and the medial laws will be used but for reference, we will not mention this on each occasion.

I) We prove first that any binary term of the list cannot be $V_{M I D}$-equivalent to a sum of at most three elements from $\operatorname{Bin}_{G}$.

1. Let $t \in \operatorname{Bin}_{G} \backslash\{x, y\}$. Assume that there exists $t_{1} \in \operatorname{Bin}_{G}$ such that $x y x+x+y+t \approx x y x+t_{1}+t \in I d V_{M I D}$.

- If $t_{1} \in \operatorname{Bin}_{G} \backslash\{x\}$, substituting $y \mapsto x y x$ in $x y x+x+y+t \approx x y x+t_{1}+t \in I d V_{M I D}$, we would have $x y x+x+x y x \approx x y x \in I d V_{M I D}$. This contradicts Lemma 2.5 (1).

- If $t_{1}=x$ substituting $x \mapsto y x y$ in $x y x+x+y+t \approx x y x+t_{1}+t \in I d V_{M I D}$, we would have $y x y+y+y x y \approx y x y \in I d V_{M I D}$. This contradicts Lemma 2.5 (1).

2. Let $t \in\{x y x, y x\}$. Assume that there exists $t_{2} \in \operatorname{Bin}_{G}$ such that $x y x+x+x y+t \approx x y x+t_{2}+t \in I d V_{M I D}$.

- If $t_{2} \in \operatorname{Bin}_{G} \backslash\{x\}$, substituting $y \mapsto x y x$ in the identity $x y x+x+x y+t \approx$ $x y x+t_{1}+t \in I d V_{M I D}$, we would have $x y x+x+x y x \approx x y x \in I d V_{M I D}$. This contradicts Lemma $2.5(1)$

- If $t_{2}=x$, since $V_{M I D}$ is solid, applying the hypersubstitution $\sigma_{x+y, y}$ to the identities $x y x+x+x y+x y x \approx x y x+x+x y x$ and $x y x+x+x y+y x \approx x y x+x+y x$, we would get $x \approx x+y+x \in I d V_{M I D}$. This contradicts the regularity property of $V_{M I D}$.

3. Let $t \in\{x y x, x y\}$. Assume that there exists $t_{3} \in \operatorname{Bin}_{G}$ such that $x y x+x+y x+$ $t \approx x y x+t_{3}+t \in I d V_{M I D}$.

- If $t_{3} \in \operatorname{Bin}_{G} \backslash\{x\}$, the substitution $y \mapsto x y x$, gives a contradiction $x y x+x+x y x \approx$ $x y x \in I d V_{M I D}$ [Lemma $\left.2.5(1)\right]$.

- If $t_{3}=x$, since $V_{M I D}$ is solid, applying the hypersubstitution $\sigma_{x+y, x}$ to the identity $x y x+x+y x+x y x \approx x y x+x+x y x$ and $x y x+x+y x+x y \approx x y x+x+x y$ we would get $x \approx x+y+x \in I d V_{M I D}$. This contradicts the regularity property of $V_{M I D}$

4. The proof is similar to 3 .

5. The proof is similar to 3 .

6 . The proof is similar to 2 .

7. The proof is similar to 1 .

II) Secondly, we prove that each term in the list of Lemma 2.8 is $V_{M I D}$-equivalent 

1. $x+x y+t, t \in\{x, x y x\}$
10. $x y x+y x+t, t \in\{x, x y x\}$
2. $x+y+t, t \in \operatorname{Bin}_{G} \backslash\{y, y x y\}$
11. $x y+x+t, t \in\{y, x y, y x y\}$
3. $x+y x+t, t \in\{x, x y x\}$
12. $x y+y x+x y$
5. $x+x y x+x$
7. $x y x+y+t, t \in \operatorname{Bin}_{G} \backslash\{y, y x y\}$
13. $x y+y+t, t \in\{x, x y, x y x\}$
8. $x y x+x y+t, t \in\{x, x y x\}$.

Notice that this enumeration corresponds exactly to that used in Lemma 2.7.

Proof of Lemma 2.9: In Lemma 2.7, we already showed that over the variety $V_{M I D}$ some sums consisting of three summands can be reduced. The same reductions are valid over $V_{B E}$ since $I d V_{M I D} \subseteq I d V_{B E}$. Therefore, using the list of all binary terms in Lemma 2.7, we have first to check whether reductions can be made by additional identities valid in $V_{B E}$.

1. One has $x+x y+y x \approx x+x y+y+y x \approx x+y+y x$ by Lemma 2.5 (1) and by Lemma 2.1 (1). The last term is considered in part 2 .

2. The identity $x+y+y x y \approx x+y x y$ arises from Lemma 2.5 (1).

3. In a similar way as we did in part 1 , we can prove $x+y x+x y \approx x+y+x y$.

4. One has $x+y x y+x \approx x+y x y+y+x \approx x+y+x$ by Lemma 2.5 (1) and Lemma $2.1(13)$.

One has also $x+y x y+x y x \approx x+y+y x y+x y x \approx x+y+x y$ by using the identity $x y x+x+x y x \approx x y x$ and Lemma 2.5 (1).

5. There is nothing to prove.

6. The identity $x y x+x+t \approx x y x+t$ is clear by Lemma $2.5(1)$.

7. Can be proved in a similar way as in part 2 .

8. In a similar way as we did in part 2 , we have $x y x+x y+y x \approx x y x+y+y x$. The last term is already considered in part 7 .

9. We have $x y x+y x y+x \approx x y x+y x y+y+x \approx x y x+y+x$ by Lemma 2.5 (1) and Lemma 2.1 (18). The last term already occurs in part 7. Also we have $x y x+y x y+x y x \approx x y x+y+y x y+x y x \approx x y x+y+x y x$ by Lemma 2.5 (1) and Lemma $2.1(7)$. The last term is considered in part 7 .

10. In a similar way as we did in part 1., one has $x y x+y x+x y \approx x y x+y+x y$. The last term is considered in part 7 .

11. Lemma 2.5 (1) shows that $x y+x+y x \approx x y+y x$ and $x y+x+x y x \approx x y+x y x$. 12. In a similar way as we did in part 1 ., we get $x y+y x+y \approx x y+x+y$ and $x y+$ $y x+x \approx x y+y+x$. The last terms already occur in part 11 . As we did in part 8 , we get $x y+y x+x y x \approx x y+y+x y x$ and $x y+y x+y x y \approx x y+x+y x y$. The last terms are already considered in part 11 .

13. Can be shown in a similar way as 11 .

14. One has $x y+x y x+x y \approx x y+x y x+x+x y \approx x y+x+x y$ by using the identity $x y x+x+x y x \approx x y x$ and Lemma 2.1 (8). see 11. for the last term.

15. On has $x y+y x y+x y \approx x y+y x y+y+x y \approx x y+y+x y$ by Lemma 2.5 (1) and Lemma 2.1 (11). The last term occurs in 13.

It is left to prove that each term in the list of Lemma 2.9 is only $V_{B E}$-equivalent to itself. This was already proved in Lemma 2.7 .

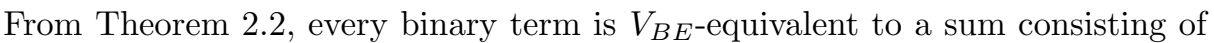
at most three summands. Using Lemma 2.3, Lemma 2.9 and the idempotency, we have the following results: 
Theorem 2.3. The universe of the free algebra with respect to $V_{B E}$ freely generated by two variables $x, y$ is $\left\{[t]_{I d V_{B E}}, t \in N^{W_{(2,2)}\left(X_{2}\right)}\left(V_{B E}\right)\right\}$, with $N^{W_{(2,2)}\left(X_{2}\right)}\left(V_{B E}\right)$ is the set consisting of elements from $\{x, x y, x y x\}$, the terms $x+t$ with $t \in$ $\operatorname{Bin}_{G} \backslash\{x\}, x y+t$ with $t \in \operatorname{Bin}_{G} \backslash\{x y\}, x y x+t$ with $t \in \operatorname{Bin}_{G} \backslash\{x y x\}$, the terms $x+t$ with $t \in \operatorname{Bin}_{G} \backslash\{x\}, x y+t$ with $t \in \operatorname{Bin}_{G} \backslash\{x y\}, x y x+t$ with $t \in \operatorname{Bin}_{G} \backslash\{x y x\}$, the terms of the list of Lemma 2.9 and all of the terms arising from the aforementioned terms by permuting the variables $x$ and $y$.

The last step of this section will be devoted to the solid variety $R A_{(2,2)}$.

Lemma 2.10. If $s_{1}+s_{2} \approx t_{1}+t_{2} \in \operatorname{IdR} A_{(2,2)}$, where $s_{i}, t_{i} \in \operatorname{Bin}_{G}$, $i \in\{1,2\}$, then $s_{1} \approx t_{1} \in \operatorname{IdR} A_{(2,2)}$ and $s_{2} \approx t_{2} \in \operatorname{IdR} A_{(2,2)}$.

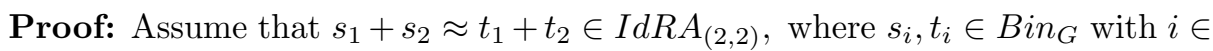
$\{1,2\}$. Since $R A_{(2,2)}$ is solid, applying the hypersubstitutions $\sigma_{x, x y}$ and $\sigma_{y, x y}$ to the previous identity, we obtain in $R A_{(2,2)}$ the identities $s_{1} \approx t_{1}$ and $s_{2} \approx t_{2}$.

Theorem 2.4. The universe of the free algebra $\mathcal{F}_{R A_{(2,2)}}\left(X_{2}\right)$ with respect to $R A_{(2,2)}$ freely generated by two variables $x, y$ is $\left\{[t]_{I d R A_{(2,2)}}, t \in N^{W_{(2,2)}\left(X_{2}\right)}\left(R A_{(2,2)}\right)\right\}$, with $N^{W_{(2,2)}\left(X_{2}\right)}\left(R A_{(2,2)}\right)=\{x, y, x y, y x, x+y, x+x y, x+y x, y+x, y+x y, y+y x, x y+$ $x, x y+y, x y+y x, y x+x, y x+y, y x+x y\}$.

Proof: Let $t \in W_{(2,2)}\left(X_{2}\right)$. Then, it is easy to verify that $t$ is $R A_{(2,2)}$-equivalent to $t_{1}+t_{2}$ where $t_{i} \in\{x, x y, y x, y\}, i=1,2$ because of Lemma 2.10 and the identities $x y z \approx x z$ as well as $x+y+z \approx x+z$ satisfied in $R A_{(2,2)}$.

Thus we have only to consider the binary terms that are sums consisting of exactly two binary terms from $\operatorname{Bin}_{G}$. Since the variety $R A_{(2,2)}$ is outermost, for $r, s, t, u \in\{x, y, x y, y x\}$ with $r \neq s$ and $u \neq t$ the equation $s+t \approx r+u$ is not an identity in $R A_{(2,2)}$. We conclude that the universe of the free algebra $\mathcal{F}_{X_{2}}\left(R A_{(2,2)}\right)$ is $\left\{[t]_{\operatorname{IdRA}_{(2,2)}}, t \in\{x, y, x y, y x, x+y, x+x y, x+y x, y+x, y+x y, y+y x, x y+x, x y+\right.$ $y, x y+y x, y x+x, y x+y, y x+x y\}\}$.

Now, we will determine the degree of each solid variety of semirings.

\section{Degrees of Solid Varieties of Semirings}

First, we give a characterisation of the trivial variety of any type $\tau$.

Theorem 3.1. Let $V$ be a variety of type $\tau=\left(n_{i}\right)_{i \in I}$ such that $n_{j} \geq 2$ for some $j \in I$. Then $V$ is a solid variety and $d_{p}(V)=1$ if and only if $V$ is trivial.

Proof: It is clear that the trivial variety $\mathcal{T}$ is solid and $d p(\mathcal{T})=1$. Let $V$ be a variety of type $\tau=\left(n_{i}\right)_{i \in I}$ such that $n_{j} \geq 2$ for some $j \in I$. Assume that $V$ is solid and $d_{p}(V)=1$. Then $\left.|P(V)|_{\sim V}|=| H y p(\tau)\right|_{\sim V} \mid=1$. Moreover, because of $n_{j} \geq 2$ for some $j \in I$, there exist at least two hypersubstitutions $\sigma_{1}$ and $\sigma_{2}$, belonging to the class of $\sigma_{i d}$, such that $\sigma_{1}\left(f_{j}\right)=x_{n_{j 1}}$ and $\sigma_{2}\left(f_{j}\right)=x_{n_{j 2}}$, with $x_{n_{j 1}} \neq x_{n_{j 2}}$. Thus we have $x_{n_{j 1}} \approx f_{j}\left(x_{1}, \cdots, n_{j}\right) \approx x_{n_{j 2}} \in I d V$. It follows that $x_{n_{j 1}} \approx x_{n_{j 2}} \in I d V$. Therefore $V$ is trivial.

The following result shows a connection between $\left.W_{(2,2)}(\{x, y\})\right|_{I d V}$ and $\left.\operatorname{Hyp}(2,2)\right|_{\sim_{V}}$ 
Lemma 3.1. Let $V$ be a variety of type $(2,2)$. The map

$$
\begin{aligned}
& \Phi:\left.\quad\left(\left.W_{(2,2)}(\{x, y\})\right|_{I d V}\right)^{2} \longrightarrow \operatorname{Hyp}(2,2)\right|_{\sim_{V}} \\
& \left([s]_{I d V},[t]_{I d V}\right) \quad \longmapsto \quad\left[\sigma_{s, t}\right]_{\sim_{V}}
\end{aligned}
$$

is bijective.

Proof: Let $s_{1}, t_{1}, s$, and $t$ four binary terms.

We have the following equivalences

$$
\begin{aligned}
\left(\left[s_{1}\right]_{I d V},\left[t_{1}\right]_{I d V}\right)=\left([s]_{I d V},[t]_{I d V}\right) & \Longleftrightarrow\left\{\begin{array}{l}
s_{1} \approx s \in I d V \\
t_{1} \approx t \in I d V \\
\sigma_{s_{1}, t_{1}}(F) \approx \sigma_{s, t}(F) \in I d V \\
\sigma_{s_{1}, t_{1}}(G) \approx \sigma_{s, t}(G) \in I d V
\end{array}\right. \\
& \Longleftrightarrow \sigma_{s_{1}, t_{1}} \sim_{V} \sigma_{s, t}
\end{aligned}
$$

It follows that $\Phi$ is well defined and is injective. By the definition $\Phi$ is onto. Therefore $\Phi$ is bijective.

Now, we have all tools to prove:

Theorem 3.2. The degrees of non trivial solid varieties of semirings with respect to proper hypersubstitution are

(1) $d_{p}\left(V_{M I D}\right)=168^{2}$

(2) $d_{p}\left(V_{B E}\right)=82^{2}$

(3) $d_{p}\left(R A_{(2,2)}\right)=16^{2}$

Proof: By Theorem 1.1, there are three non trivial solid varieties of semirings, namely $V_{M I D}, V_{B E}$ and $R A_{(2,2)}$. For any solid variety $V$ of semirings, we have $d p(V)=|P(V)| \sim_{V}|=| \operatorname{Hyp}(2,2)\left|{ }_{\sim_{V}}\right|$.

But Lemma 3.2 ensures that $|H y p(2,2)|_{\sim_{V}}|=|\left(\left.W_{(2,2)}(\{x, y\})\right|_{I d V}\right)^{2} \mid=\left(\left|W_{(2,2)}(\{x, y\})\right|_{I d V} \mid\right)^{2}$.

Therefore, using Theorem 2.1, Theorem 2.3 and Theorem 2.4 we have the results. This means, if $V$ is a solid variety of semirings, then $d_{p}(V) \in\left\{1,168^{2}, 82^{2}, 16^{2}\right\}$.

\section{REFERENCES}

[1] Denecke, K., Hounnon, H., Solid Varieties of Normal ID-Semirings, General Algebra and Discrete Mathematics, Proceedings of the 59th Workshop on General Algebra, 15th Conference for Young Algebraists, Potsdam 2000, Shaker Verlag Aachen (2000), 25-40.

[2] Denecke, K., Hounnon, H., Solid Varieties of Semirings, Proceedings of the International Conferenc on Semigroups, Braga (Portugal) 1999, World Scientific (2000), 69-86.

[3] Denecke, K. and Hounnon, H., All solid varieties of semirings, Journal of Algebra 248 (2002), 107-117.

[4] Denecke, K., Koppitz, J., Srithus, K., N-fluid varieties, Scientiae Mathematicae Japonicae 65, No. 1 (2007), 1-19: e-2006, 1025-1034.

[5] Denecke, K. Koppitz, J., Srithus, K., The Degree of Proper Hypersubstitutions, Scientiae Mathematicae Japonicae Online e-2007, 301-314.

[6] Denecke, K., Srithus, K., Binary Relations on the Monoid of $V$-proper Hypersubstitutions, Discussiones Mathematicae, General Algebra and Applications 26 (2006), 233-251.

[7] Denecke, K., Wismath, S. L., Hyperidentities and Clones, Gordon and Breach Science Publishers (2000).

[8] Graczyńska, E. On normal and regular identities and hyperidentities, Proceedings of the V Universal Algebra Symposium, Universal and Applied Algebra, Turawa, Poland, Word Scientific (1989), 107-135.

[9] Graczyńska, E. and Schweigert, D. Hypervarieties of a given type, Algebra Universalis, 27 (1990), 305-318. 
[10] Hounnon, H., Hyperidentities in Semirings and Applications Shaker Verlag, Aachen (2002)

[11] Płonka, J., Proper and inner hypersubstitutions of varieties, Proceedings of the International Conference: Summer School on General Algebra and Ordered Sets, Palacky University Olomouc (1994), 106-115.

[12] R. McKenzie, G. McNulty and W.F. Taylor, Algebras, Lattices Varieties Vol 1, 1987 Inc. Belmonts Califormia.

[13] Srithus, R. Algebras Derived by Hypersubstitutions, PhD thesis, Potsdam University, Germany (2008)

Facultedes Sciences et Techniques Universite D'Abomey-Calavi 01 BP 526 ReP. DU BENIN

E-mail address: hiph14@yahoo.fr

University of Potsdam Am Neuen Palais 10, House 914469 Potsdam

E-mail address: klausdenecke@hotmail.com 